\title{
Non-additive properties of finite 1D Ising chains with long-range interactions
}

\author{
S. S. Apostolov, Z. A. Mayzelis, O. V. Usatenko** and V. A. Yampol'skii \\ A. Ya. Usikov Institute for Radiophysics and Electronics \\ Ukrainian Academy of Sciences, 12 Proskura Street, 61085 Kharkov, Ukraine \\ We study the statistical properties of Ising spin chains with finite (although ar- \\ bitrary large) range of interaction between the elements. We examine mesoscopic \\ subsystems (fragments of an Ising chain) with the lengths comparable with the in- \\ teraction range. The equivalence of the Ising chains and the multi-step Markov \\ sequences is used for calculating different non-additive statistical quantities of a \\ chain and its fragments. In particular, we study the variance of fluctuating magne- \\ tization of fragments, magnetization of the chain in the external magnetic field, etc. \\ Asymptotical expressions for the non-additive energy and entropy of the mesoscopic \\ fragments are derived in the limiting cases of weak and strong interactions. \\ PACS numbers: 05.40.-a, 02.50.Ga, 05.50.+q
}

\section{INTRODUCTION}

One of the basic postulates of the traditional statistical physics is the assumption that the range of particle interaction is small compared to the system size. If this condition does not hold the internal and free energy, entropy, and other physical quantities are no longer additive. Due to this fact, there are no straightforward definitions of the temperature, entropy, etc. The statistics of the systems with long-range interaction are not Gibbsian; Boltzman's relationship between the entropy and the statistical weight is no longer valid; the fluctuations of sums of random quantities do not obey the Gaussian statistics.

Systems with long-range interaction are common in physics [1]. Amongst them are: gravitating systems [2], electrically charged systems [3], magnets with dipolar interactions [4],

\footnotetext{
*usatenko@ire.kharkov.ua
} 
etc. An important physical example of such class of systems is the Ising spin chain with long-range interaction (see, e.g., [5]).

The long-range interaction leads to the correlations between the elements of the system, which is the reason for the appearance of non-Gaussian statistics. Such non-Gaussian distributions (induced by the long-range correlations) are found in various fields of science, e.g., in linguistics (distribution of "words" in a given text [6, 7, 8]), biology (the distribution of nucleotides in DNA sequences [9]), computer science (forward error correction codes [10]), economics (the distributions in financial markets [11]), sociology, physiology, seismology.

One of the most interesting phenomena in the long-range correlated systems is the phenomenon of phase transition. The existence of phase transitions is of great importance for different sciences, for example, biology in the problem of DNA melting [12, 13]. Dyson [14], Ruelle [15], Thouless [16] found different conditions for existence of the phase transition in the Ising chain with the infinite scale of interaction.

The usual objects for studying the effects of long-range correlations are the non-extensive thermodynamic systems [17] where both interaction range and the system length are macroscopically large. However, the mesoscopic long-range correlated systems, which are not large enough to consider the thermodynamic limit, have not been studied thoroughly. The statistical properties of such systems are very similar to the properties of the infinite non-extensive thermodynamic systems. In the present paper, we consider the finite-size subsystems (fragments) of an infinite Ising chain as examples of mesoscopic correlated systems. We study the Ising chain with the Hamiltonian,

$$
\mathcal{H}=-\sum_{0<j-i<N} \varepsilon(j-i) s_{i} s_{j}-H \sum_{i} s_{i}
$$

where the spin variable $s_{i}$ takes on two values, -1 and $1, \varepsilon(r)>0$ is the exchange integral of the ferromagnetic coupling, and $H$ is the external magnetic field. Below, for the simplicity, we assume $\varepsilon(r)=0$ for $r>N$. The range $N$ of spin interaction is finite although arbitrary. The chain is assumed to be in the thermodynamical equilibrium with the Gibbs thermostat of temperature $T$ (unless otherwise mentioned). Such a system is obviously extensive one, and the usual thermodynamics can be applied to the system as a whole. There is no spontaneous magnetization in such a chain due to the finiteness of the particle interaction range. Nevertheless, its mesoscopic subsystems are of interest because of their non-additive statistical properties. The aim of our work is to calculate different statistical quantities for 
an mesoscopic subsystem of length $L$, that can be of the order of the interaction length $N$.

Thus, we study an Ising chain with the finite range of interaction, but the lengths of the subsystems are also finite. We show that energy and entropy of the fragments of the Ising chain are non-additive quantities. We calculate the root-mean-square (RMS) value of fluctuation of the magnetization which does not scale as $\sqrt{L}$ (the number of spins in a fragment), unlike the case of short-range interactions. We suggest a way to calculate statistical quantities for the finite subsystems in contact with both the external Gibbs thermal bath of temperature $T$ and the rest of the Ising chain playing a role of additional thermostat.

For mesoscopic systems, an important problem is to introduce the appropriate statistical ensemble correctly, because different statistical ensembles are not equivalent for such systems (see, e.g., Ref. 18). These canonical ensembles are equivalent in the thermodynamical limit only. In the present paper, we deal with an ensemble of the finite subsystems that are the parts of an infinite Ising chain.

We calculate the statistical properties of the Ising chains using their equivalence with the binary $N$-step Markov chains. The multi-step Markov chain is the sequence where symbols, say \pm 1 , are generated by means of the following procedure. One starts from arbitrary $N$ symbols, than the subsequent symbols are generated with the probability that depends on the values of the preceding $N$ symbols only and is independent of the farther symbols,

$$
P\left(s_{i}=s \mid T_{i, \infty}^{-}\right)=P\left(s_{i}=s \mid T_{i, N}^{-}\right)
$$

Here $T_{i, L}^{-}$is a set of $L$ sequential symbols $\left(s_{i-L}, s_{i-L+1}, \ldots, s_{i-1}\right)$. The statistical properties of such chains were studied in detail in Refs. 7, 19, 20, 21, 22, 23.

Statistical equivalence of the Ising and Markov chains is widely known (see, e.g., Refs. 24, 25). However, these References define Markov chains not via the "one-sided" conditional probability function Eq. (2), depending on the values of the preceding symbols, but rather via the "two-sided" one. The definition of the chain with one-sided conditional probability function is more convenient for numerical simulations of the chain as well as for obtaining analytic results. Reference 26 demonstrated the equivalence of the two mentioned views on Markov chains.

The paper is organized as follows. In the second Section we show how to find the Markov chain corresponding to the Ising chain under consideration. Using this correspondence rule we calculate the RMS value of the fluctuating part of magnetization for the chain fragments 
of the length $L$ in the absence of magnetic field. We also find magnetization of the chain in the presence of the magnetic field. The third Section introduces the method for calculating the statistical quantities of the fragments of Ising chain, in particular, the internal and total energy, and entropy. All these quantities are non-additive, i.e., they do not scale as the fragment length $L$. We obtain analytical results for the two limiting cases of low and high temperatures.

\section{MAGNETIC PROPERTIES OF ISING CHAIN}

First, we find a one-sided conditional probability function of an $N$-step Markov chain, Eq. (2), that is statistically equivalent to the Ising chain with Hamiltonian (1). Then we use this property to find the magnetization of the Ising chain for the case of weak interactions (compared to the temperature). Finally, we study another limiting case of strong interactions.

\section{A. Equivalence of Markov and Ising chains}

The conditional probability of a spin to have a definite value, say 1 , given that the values of all other spins in the chain are fixed, is independent of the values of the spins separated by the distances larger than $N$ (see, e.g., Refs. 24, 25) and reads

$$
P\left(s_{i}=1 \mid T_{i, \infty}^{-}, T_{i, \infty}^{+}\right)=P\left(s_{i}=1 \mid T_{i, N}^{-}, T_{i, N}^{+}\right)
$$

Here $T_{i, L}^{+}$is the $L$-tuple, $\left(s_{i+1}, s_{i+2}, \ldots, s_{i+L}\right)$. The probability function in Eq. (3) is given by the Gibbs formula, and for the Hamiltonian (1) it has the following form:

$$
P\left(s_{i}=1 \mid T_{i, N}^{-}, T_{i, N}^{+}\right)=\left(1+\exp \left(-\frac{2 H}{T}-\sum_{r=1}^{N} \frac{2 \varepsilon(r)}{T}\left(s_{i-r}+s_{i+r}\right)\right)\right)^{-1}
$$

Expression (4) for the two-sided conditional probability function can be derived by the method proposed in Section III. It can be shown that the Metropolis scheme [27] also yields the same expression (4) for the conditional probability.

Reference 26 analytically proved that the chain defined by a two-sided conditional probability function (3) is equivalent to the $N$-step Markov chain defined by Eq. (2). The relation 
between the two-sided and one-sided Markov conditional probability functions reads

$$
P\left(s_{i}=s \mid T_{i, N}^{-}, T_{i, N}^{+}\right)=\frac{\prod_{\substack{r=0 \\ s_{i}=1}}^{N} P\left(s_{i+r} \mid T_{i+r, N}^{-}\right)}{\prod_{\substack{r=0 \\ s_{i}=1}}^{N} P\left(s_{i+r} \mid T_{i+r, N}^{-}\right)+\prod_{\substack{r=0 \\ s_{i}=-1}}^{N} P\left(s_{i+r} \mid T_{i+r, N}^{-}\right)} .
$$

Unfortunately, this relation is quite cumbersome and can hardly be applied to the analytical study of Ising chains in the general case. Nevertheless, it allows one to calculate numerically the one-sided conditional probability function for the $N$-step Markov chain. Thus, to attain equilibrium state of the spin chain, we can generate the Markov chain according to the algorithm described in the Introduction, instead of using the Metropolis scheme.

\section{B. Magnetization of the Ising chain with weak interaction}

In order to use Eqs. (44) and (5) for the analytical study of Ising chains we consider the case of weak interaction compared to the temperature,

$$
\sum_{r=1}^{N} \varepsilon(r) \ll T .
$$

Expanding Eq. (44) over the small parameter $\sum_{r=1}^{N} \varepsilon(r) / T$ and using Eq. (5) we obtain the one-sided conditional probability function. In the first approximation, it takes the additive form,

$$
P\left(s_{i}=1 \mid T_{i, \infty}^{-}\right)=\frac{1+\bar{s}}{2}+\sum_{r=1}^{N} F(r)\left(s_{i-r}-\bar{s}\right) .
$$

Here the function $F(r)$ and $\bar{s}$ (the average value of $s_{i}$ ) are determined as follows,

$$
F(r)=\frac{\varepsilon(r)}{2 T} \cosh ^{-2} \frac{H}{T}, \quad \bar{s}=\left(1+4 \sum_{r=1}^{N} F(r)\right) \tanh \frac{H}{T} .
$$

The Markov chain with additive conditional probability function, Eq. (7), is referred to as the additive Markov chain. The function $F(r)$ is referred to as the memory function [7]. Equation (8) reflects the obvious fact that an increase of the temperature leads to an increase of disorder and to decrease of correlations in the chain. It should be noted that the interaction 
range $N$ in Eqs. (7) and (8) can be taken as $\infty$, if the series $\sum_{r}|F(r)|$ converges and the value of $P$ in Eq. (7) satisfies the inequality $0<P\left(s_{i}=1 \mid T_{i, \infty}^{-}\right)<1$.

Thus, the Ising chain in the equilibrium is equivalent to the additive Markov chain. For the case of weak interaction between the spins, the corresponding memory function $F(r)$ is proportional to the energy $\varepsilon(r)$. The memory function of the additive Markov chain is related to its pair correlation function $K(r)$,

$$
K(r)=\overline{s_{i} s_{i+r}}-\bar{s}^{2}
$$

by the recurrence equation [28],

$$
K(r)=\sum_{r^{\prime}=1}^{N} 2 F\left(r^{\prime}\right) K\left(r-r^{\prime}\right), \quad r=1,2, \ldots
$$

The overline $\ldots$ in Eq. (9) denotes statistical averaging. In the limiting case of small $F(r)$, Eq. (10) yields approximately,

$$
K(r) \approx 2 F(r), \quad r=1, \ldots, N
$$

\section{Fluctuations of magnetization in the absence of magnetic field}

We now consider an Ising chain with weak but long-range interaction in the absence of the magnetic field. As mentioned above, the averaged magnetization equals to zero in this case. However, the root-mean-square (RMS) value of the magnetization of a chain segment with length $L$,

$$
M_{\mathrm{RMS}}(L)=\left(\overline{\left(s_{i+1}+s_{i+2}+\cdots+s_{i+L}\right)^{2}}\right)^{1 / 2},
$$

is not zero and is determined by the correlation function of the chain,

$$
M_{\mathrm{RMS}}(L)=\left(L+2 \sum_{r=1}^{L-1}(L-r) K(r)\right)^{1 / 2} .
$$

Using Eqs. (8) and (11), we derive the relation between the RMS of magnetization and the interaction energy,

$$
M_{\mathrm{RMS}}(L)=\sqrt{L}+\frac{1}{T \sqrt{L}} \sum_{i=1}^{L-1}(L-i) \varepsilon(i) .
$$

Here we used that $\varepsilon(r)=0$ for $r>N$, thus the range $N$ of interaction does not appear explicitly. 
The interaction between spins leads to the deviation of the RMS of magnetization from the value $\sqrt{L}$ occurring in the non-interacting chain. If this deviation is small, as we assume here, the distribution function of magnetization is close to the Gaussian form with the variance $M_{\mathrm{RMS}}^{2}(L)$.

\section{Magnetization in the external magnetic field}

If the magnetic field $H$ is applied, the averaged value $\mu$ of magnetization (per spin) is no longer zero. In the assumption of weak interactions, Eq. (66), $\mu$ is equal to the averaged value of the spin $\bar{s}$, Eq. (8),

$$
\mu=\bar{s}=\left(1+\frac{2}{T} \cosh ^{-2} \frac{H}{T} \sum_{r=1}^{N} \varepsilon(r)\right) \tanh \frac{H}{T} .
$$

In the nearest-neighbor approximation $(N=1)$, this equation coincides with the hightemperature asymptotics of the well-known expression for the magnetization,

$$
\mu=\operatorname{sign} H\left(1+\frac{\exp (-4 \varepsilon / T)}{\sinh ^{2}(H / T)}\right)^{-1 / 2} .
$$

Here $\varepsilon=\varepsilon(1)$ is the single exchange integral for the nearest neighbors.

The results of numerical simulations for the Ising chain with weak interaction are given in the left panel of Fig. 1. Here we present the dependence of the magnetization on the value of magnetic field. The inset shows the dependence of the RMS of magnetization on the segment length $L$ in the absence of the magnetic field. The numerical points are very close to the corresponding analytical curves (solid lines) determined by Eqs. (15) and (14).

\section{Magnetic properties of the Ising chain in the low-temperature limit}

In the previous subsection, we have used the statistical equivalence of the Markov and Ising chains for the calculation of the magnetization in the case of high temperatures. Here we examine the opposite limiting case of low temperatures. The corresponding inequality imposed on the temperature $T$, the interaction-energy function $\varepsilon(r)$, and magnetic field $H$ will be given below (see Eq. (19)). In this case, Eq. (44) defines a non-additive Markov chain (see Ref. 26 for details). Since finding the correlation function for such chains brings about considerable technical difficulties, we examine the required statistical properties of the Ising chain directly from Eq. (44). 


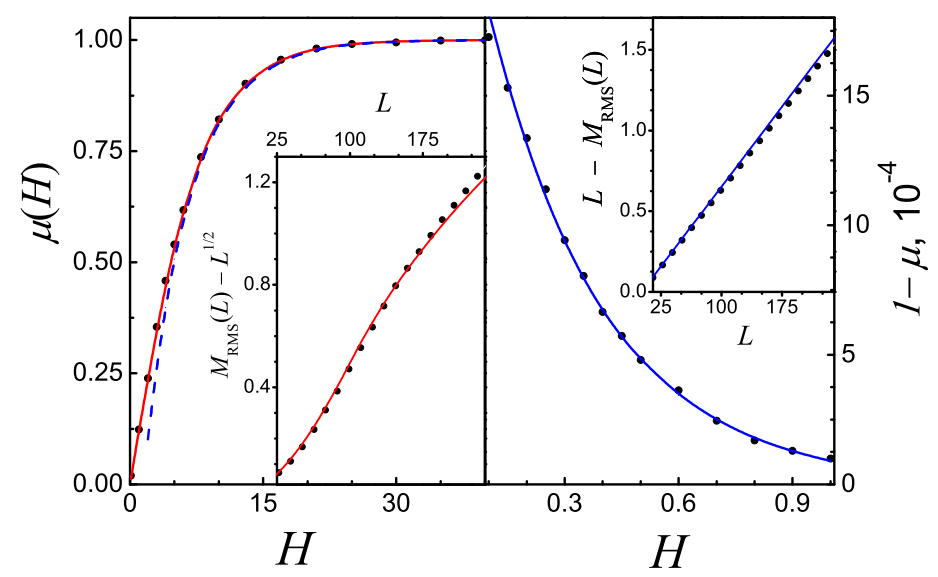

FIG. 1: (Color online) The magnetization $\mu$ of the chain per spin versus the magnetic field $H$. Left panel $(T=10)$ : solid and dashed curves correspond to the high-temperature, Eq. (15), and low-temperature, Eq. (22), limits, respectively. Right panel $(T=0.6)$ : solid curve corresponds to low-temperature, Eq. (22), limit. The RMS magnetization in the absence of magnetic field versus length $L$ of a segment is presented in the insets. The dots correspond to the results of the numerical simulations. The function $\varepsilon(r)$ is step-like, $\varepsilon(r)=1 / 100$ for $r=1,2, \ldots, 100$ and $\varepsilon(r)=0$ for $r>100$.

\section{Statistical properties of strongly correlated Ising spin chain}

In the case of low temperatures, the conditional probability determined by Eq. (44) is close either to zero or one. Indeed, due to strong correlations, the chain consists of large domains predominantly occupied by the same symbols. It is evident that, once strong enough magnetic field is applied, the chain will mostly consist of the same symbols.

For definiteness, we consider the case when almost all symbols in a chain are 1 (the case of $H>0$ ). In other words, the probability to find the spin -1 at a given place of the chain is small. For further convenience, it is suitable to introduce the parameter $\delta$,

$$
\delta=\exp \left(-4 \epsilon_{1} / T\right) \ll 1, \quad \epsilon_{1}=\sum_{r=1}^{N} \varepsilon(r)+H / 2 .
$$

According to Eq. (4), this parameter is a ratio of the probability to find the $(2 N+1)$-tuple with only one spin -1 in the center to the probability of occurring the $(2 N+1)$-tuple with all spins 1 . Due to the correlations, the probability to have the $(2 N+1)$-tuple with one more spin -1 at distance $k$ from the central spin -1 differs from $\delta^{2}$ and is equal to $\delta^{2} \sigma_{k}$ 
with

$$
\sigma_{k}=\exp (4 \varepsilon(k) / T) \geqslant 1
$$

Further, due to Eq. (4), three spins -1 (at successive distances $k$ and $l$ ) occur even less frequently, with the probability $\delta^{3} \sigma_{k} \sigma_{l} \sigma_{l+k}$. The corresponding probabilities for four and more spins -1 can be found in a similar way.

We use the following assumptions for the parameters of the system:

$$
\delta \sigma_{1} \sum_{l=1}^{N} \sigma_{l}^{2} \ll(1+\exp (-2 H / T))^{-2 N} .
$$

This inequality states that the probabilities to have a $(2 N+1)$-tuple with three or more spins -1 is negligible compared to that with just two spins -1 . Inequality (19) holds only for the case of monotonously decreasing functions $\varepsilon(r)$. In general case, we should replace $\sigma_{1} \sum \sigma_{l}^{2}$ in Eq. (19) by the maximum value of $\sigma_{k} \sum \sigma_{l} \sigma_{l+k}$.

Inequality (19) can be considerably simplified under some suppositions. For example, if function $\varepsilon(r)$ is rapidly decreasing $(\varepsilon(1) \gg \varepsilon(2) \gg \ldots)$ and $H>4 \varepsilon(1)$ we obtain

$$
T \ll H-4 \varepsilon(1) .
$$

For chains with approximately step-like function $\varepsilon(r)(\varepsilon(r) \approx \varepsilon(1)$ for $r \leq N$ and $\varepsilon(r)=0$ for $r>N$ ), we have

$$
T \ll N \varepsilon(1)+H / \ln N .
$$

Thus, Eq. (19) means that the temperature $T$ should be sufficiently small compared with $H$ or $\varepsilon(i)$.

It should be noted that inequalities (19) and (6) can be satisfied simultaneously. This is the case when $\sum \varepsilon(r) \ll T \ll H / N$. Then, the results of subsection $\amalg B$ coincide with those of this subsection (Eq. (15) transforms to Eq. (22)).

\section{Magnetization of the Ising chain}

Using Eqs. (44), (17), and (18), we derive average magnetization $\mu$ per spin in the presence of the magnetic field $H>0$,

$$
\mu=\bar{s}=1-2 \delta+2 \delta^{2}-4 \delta^{2} \sum_{k=1}^{N}\left(\sigma_{k}-1\right) .
$$


For strong enough magnetic fields, $\exp (-H / T) \ll 1$, this expression is also valid in the hightemperature limit Eq. (6). Moreover, for chains with nearest-neighbor interaction $(N=1)$, it coincides with the general formula (16) in the main and first approximations with respect to the parameter $\exp (-(H-4 \varepsilon) / T) \ll 1$.

If the magnetic field is not applied, the averaged magnetization is equal to zero. However, the RMS of magnetization, determined by the pair correlation function $K(r)$, is non-zero. In the case of low temperatures, $K(r)$ is close to one. For $r \leqslant N$,

$$
K(r) \approx 1-4 \delta
$$

Therefore, the RMS of magnetization of segments with length $L \ll L_{0}$ is equal to $L$ in the main approximation with respect to $\delta$, contrary to the square-root dependence $\sqrt{L}$ valid in the high-temperature limit. Here $L_{0} \gg N / \delta$ is the characteristic length of the domains consisting of the same symbols, 1 or -1 . Using Eqs. (13) and (23), we obtain,

$$
M_{\mathrm{RMS}}(L)=L-2(L-1) \delta,
$$

The results of numerical study of strongly correlated chains are shown in Fig. 1. As in the case of weak interaction, we present calculations of the RMS of magnetization for the segment of length $L$ in the absence of the magnetic field (inset in the right panel) and the averaged magnetization as the function of the magnetic field $H$ (solid curve in the left panel and dashed curve in the right panel). All the results of numerical simulations are close to the theoretical predictions. As mentioned above, two asymptotics Eqs. (15) and (22) coincide for the strong enough magnetic fields at $\sum \varepsilon(r) \ll T$. This is clearly seen in the left panel.

Thus, we have studied the magnetization and its RMS for the Ising chains in the two limiting cases of high and low temperatures. These quantities are determined by the statistical properties of the chains, particularly, by the correlation function. Not only the magnetization, but all the other statistical properties of the Ising chain can be studied using the equivalence of the Ising and Markov chains (see Ref. 21). In the next section, we propose a general way of calculating the statistical quantities of the Ising chains with long-range spin interaction. 


\section{TOTAL AND INTERNAL ENERGY AND ENTROPY OF ISING CHAIN SEGMENTS}

Here we present an approach to calculate any statistical quantity (energy, entropy, etc.) for a subsystem of arbitrary length $L$ (a segment of $L$ sequential spins in the Ising chain). This mesoscopical subsystem, denoted by $M$, interacts with the thermostat and with the rest of the chain. We denote also two "border" subsystems of the length $N$ by $B$. All the other spins, except for $M$ and $B$, are denoted by $R$ :

$$
\underbrace{\ldots s_{i-N}}_{R} \underbrace{s_{i-N+1} \ldots s_{i}}_{B} \underbrace{s_{i+1} \ldots s_{i+L}}_{M} \underbrace{s_{i+L+1} \ldots s_{i+L+N}}_{B} \underbrace{s_{i+L+N+1} \cdots}_{R}
$$

The total energy of the chain is not equal to the sum of the internal energies of subsystems $M$ and $(B+R)$. So, the long-range interaction between $M$ and $(B+R)$ makes it impossible to use directly the standard methods of statistical physics for calculation the statistical quantities for segment $M$.

In order to find the condition of equilibrium, we introduce the statistical ensemble of the chains. We choose the subensemble of chains with fixed border regions $B$ (but with varying $M$ and $R$ ). For each of $2^{2 N}$ such subensembles, the subsystem $B$ plays the role of a separating wall. If the ensemble is in the equilibrium state, the total energy of $(M+R)$ (that includes the sum of internal energies of $M$ and $R$, and energy of their interaction with $B)$ is constant for every subensemble. Thus, we can use the equilibrium condition between $M$ and $R$ :

$$
\frac{\partial \ln W_{M}\left(E_{M} \mid B\right)}{\partial E_{M}}=\frac{\partial \ln W_{R}\left(E_{R} \mid B\right)}{\partial E_{R}}=\frac{1}{T},
$$

where $W_{M}\left(E_{M} \mid B\right)$ and $W_{R}\left(E_{R} \mid B\right)$ are the statistical weights of subsystems $M$ and $R$ with total energies $E_{M}$ and $E_{R}$, with the borders $B$ fixed. We refer to statistical weights $W_{M}\left(E_{M} \mid B\right)$ and $W_{R}\left(E_{R} \mid B\right)$ as the conditional statistical weights. It should be emphasized that these weights are not mesoscopical quantities because of their dependence on the microscopic states of borders $B$. If the system is in the thermal contact with the Gibbsian thermostat, the condition of equilibrium between the thermostat and segment $M$ sets the temperature of $M$ equal to $T$. This temperature is obviously the same for every subensemble with fixed $B$. Thus, the averaged temperature of the subsystem $M$ is also $T$. 
Note that the external thermostat is not necessary for establishing the temperature $T$ of the mesoscopical segment $M$. The role of a thermostat can be played by the subsystem $R$. Indeed, the temperature determined by Eq. (25) does not depend on values of spins in $B$ (because the statistics of infinite subsystem $R$ can not depend on the microstate of finite subsystem $B$ ). This means that, for all the subensembles, the temperature of $M$ is the same even in the absence of the external thermostat. In this case, the temperature in the chain is determined by its initial state (that can be nonequilibrium one).

It is very important that the distribution function of the segment $M$ over different microstates within a subensemble with fixed borders $B$ is Gibbsian (though the segment $M$ itself is not a Gibbsian system). Therefore, within every subensemble, we can introduce a conditional statistical quantity $Q_{M}(\mid B)$ for the segment $M$. The actual quantity $Q_{M}$ is the conditional one, averaged over the subensembles with different borders $B$ :

$$
Q_{M}=\left\langle Q_{M}(\mid B)\right\rangle_{B}
$$

With this method for calculating the statistical quantities, we do not need to find the distribution function for the segment $M$ over different microstates in the whole ensemble. Note also that Eq. (26) can be considered in the thermodynamical limit $L \rightarrow \infty$. However, we focus our attention on the mesoscopical segments $M$ with finite length $L$.

The conditional entropy can be introduced as the logarithm of the conditional statistical weight: $S_{M}\left(E_{M} \mid B\right)=\ln W_{M}\left(E_{M} \mid B\right)$. Equality $d S_{M}\left(E_{M} \mid B\right)=d E_{M}(T \mid B) / T$ is fulfilled for the conditional quantities $S_{M}(\mid B)$ and $E_{M}(\mid B)$. Meanwhile, such a relation is not valid for the averaged entropy $S_{M}\left(E_{M}\right)=\left\langle S_{M}\left(E_{M} \mid B\right)\right\rangle_{B}$ and total energy $E_{M}(T)=\left\langle E_{M}(T \mid B)\right\rangle_{B}$.

Note that the presented method for calculating the statistical quantities is rather general and can be applied, e.g., to the internal energy of a segment $M$ that can be measured experimentally, or to the probability for some spin to take on the definite value under the condition of fixed environment. Considering one spin $s_{i}$ as a subsystem $M$ and the conditional probability $P$ as a quantity $Q$ in the above-mentioned method, one arrives at Eq. (41).

Now we apply our approach to calculation of the total and internal energy and entropy of the segments of length $L$ for the limiting cases of high and low temperatures. 


\section{A. High-temperature limit}

The total energy of a segment $M$ with length $L$ for prescribed configuration of $M$ and $B$ is expressed via products of pairs of spins,

$$
E(L \mid M, B)=-H \sum_{j=i+1}^{i+L} s_{j}-\sum_{j=i+1}^{i+L} \sum_{\substack{k=j-N \\ k \neq j}}^{j+N} \varepsilon(|k-j|) s_{k} s_{j}+\sum_{\substack{j, k=i+1 \\ j<k}}^{i+L} \varepsilon(k-j) s_{k} s_{j} .
$$

The actual total energy is the conditional energy averaged over the different configurations of $M$ and $B, E(L, T)=\overline{E(L \mid M, B)}$. Along with the total energy $E(L, T)$, the segment $M$ can be characterized by its internal energy $E^{\text {in }}(L, T)=\overline{E^{\text {in }}(L \mid M, B)}$ specifying the interaction between the spins in $M$ only,

$$
E^{\mathrm{in}}(L \mid M, B)=-H \sum_{j=i+1}^{i+L} s_{j}-\sum_{\substack{j, k=i+1 \\ j<k}}^{i+L} \varepsilon(k-j) s_{k} s_{j}
$$

This quantity can be measured experimentally and thus is of interest for study.

The second term in Eq. (27) accounts the interaction of each spin of the segment $M$ with $2 N$ surrounding spins. The internal interaction energy is accounted twice in this term. Thus, the third term in Eq. (27) subtracts the corresponding extra summands.

In the high-temperature limit, the total and internal energy can be calculated via the pair correlation function $K(r)$ with $r \leqslant N$, without the need for the conditional energies. Using Eqs. (8) and (11), we arrive at

$$
\begin{gathered}
E(L, T)=-\left(\epsilon_{2} L-\sum_{r=1}^{L-1}(L-r) \varepsilon^{2}(r)\right) / T, \\
E^{\mathrm{in}}(L, T)=-\left(\sum_{r=1}^{L-1}(L-r) \varepsilon^{2}(r)+H^{2} L\right) / T,
\end{gathered}
$$

with

$$
\epsilon_{2}=2 \sum_{r=1}^{N} \varepsilon^{2}(r)+H^{2}, \quad H \ll T .
$$

If the segment length $L$ is much greater than the memory length $N$ this expression yields additive energies, $E(L, T) \propto L$. For the opposite limiting case, $L \ll N$, we get:

$$
E(L, T) \approx-\epsilon_{2} L / T+\varepsilon^{2}(1) L^{2} / 2 T, \quad E^{\text {in }}(L, T) \approx-\varepsilon^{2}(1) L^{2} / 2 T-H^{2} L / T .
$$

Here we suppose that $\varepsilon(i) \approx \varepsilon(1)$ for $i \lesssim L$. 


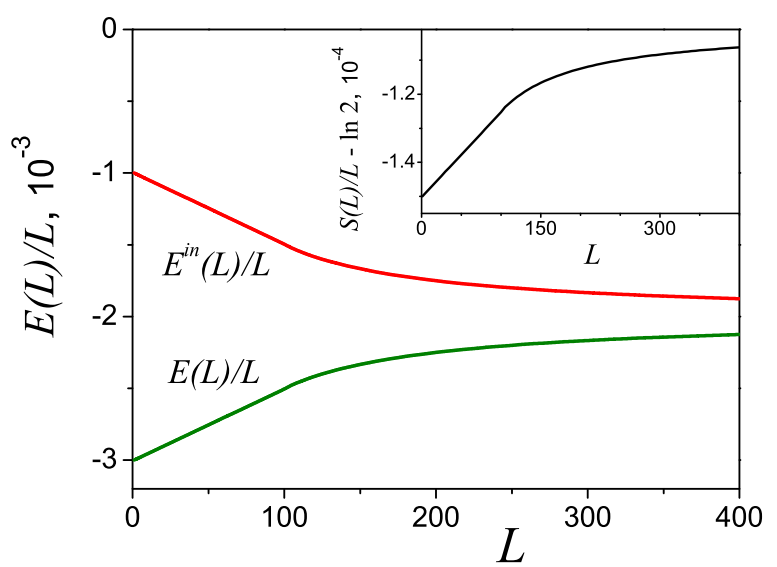

FIG. 2: (Color online) The dependences of specific non-additive energy $E(L) / L$ and $E^{\text {in }}(L) / L$ on the length $L$ of a segment for the step-like function $\varepsilon(r)=1 / 100, \quad r \leq 100$. The specific nonadditive entropy $S(L) / L$ versus length $L$ is presented in the inset. Other parameters are: $T=10$, $H=0.1$.

The non-additive energy is expressed in terms of the pair correlation function only. This is not correct for other statistical quantities, e.g., the entropy of the segment $M$. Formally, in order to find the entropy, one should calculate all the conditional entropies by integrating the equation $d S(L, T \mid B)=d E(L, T \mid B) / T$, and averaging the result over all realizations of the borders $B$. However, at high temperatures, to a first order in the small parameter $\epsilon_{1} / T$, we can change the order of these operations and calculate the averaged entropy by integrating the formula $d S(L, T)=d E(L, T) / T$ written for the averaged energy. A constant of integration is determined from the condition of complete randomization at high temperatures, $S(L, T \rightarrow \infty) \rightarrow \ln \left(2^{L}\right)$. Thus, we obtain

$$
S(L, T)=L \ln 2+E(L, T) / 2 T
$$

with $E(L, T)$ given by Eq. (29).

Expressions (29)-(33) describe the non-additive dependence of energy and entropy of a segment $M$ on its length $L$. Note that the energy is non-additive in the main approximation in the parameter $\epsilon_{1} / T$, while the non-additivity of the entropy appears in the first-order correction only. The dependences of the non-additive energy and entropy on $L$ are presented in Fig. 2 for the step-like function $\varepsilon(r)$.

Knowing the energy and entropy we can find some other statistical quantities. For example, at high temperatures the heat capacity can be determined in a similar way as for the 
entropy. One can use the classical formula $C(L, T)=T d S(L, T) / d T$ with averaged entropy given in Eq. (33) and obtain the equation

$$
C(L, T)=-E(L, T) / T=\left(\epsilon_{2} L-\sum_{r=1}^{L-1}(L-r) \varepsilon^{2}(r)\right) / T^{2} .
$$

This formula is valid for the case of high temperatures in the main approximation only. In the general case, $C(L, T)$ is not determined by equation $C(L, T)=T d S(L, T) / d T$. This relation holds for the conditional quantities only.

For the case of high temperatures, we calculated the averaged non-additive energy and entropy without using the conditional ones. In the opposite limiting case of low temperatures, it is necessary to calculate the conditional quantities as well.

\section{B. Low-temperature limit}

In this subsection, we assume that inequality Eq. (19) is fulfilled. Let us find the averaged values of the total and internal energy and entropy of a segment $M$ consisting of $L$ spins. These are close to their minimal values taken at $T=0$ when all spins in segment $M$ (as well as in borders $B$ ) are oriented along the magnetic field $H$. For positive magnetic fields, $H>0$, we have

$$
E_{\min }(L)=-\left(\epsilon_{1}+H / 2\right) L-\sum_{r=1}^{N} r \varepsilon(r), \quad E_{\min }^{\mathrm{in}}(L)=-\left(\epsilon_{1}+H / 2\right) L+\sum_{r=1}^{N} r \varepsilon(r) .
$$

For simplicity, we consider here and below the segments with $L \geqslant N$.

According to the Nernst theorem, the entropy in the zeroth approximation is $S(L, T=$ $0)=0$. Now we seek the first non-additive corrections to $E, E^{\text {in }}$, and $S$. Following the proposed approach, we have to find, as a first step, the conditional total energy and entropy for all the configurations of borders $B$ :

i) The most probable configuration of borders $B$ is $B_{0}$ when they consist of spins +1 only. The corresponding probability $P\left(B_{0}\right)$ is approximately equal to $1-2 N \delta$. The conditional 
total energy and entropy for this case can be found by virtue of Eqs. (17), (18), and (27):

$$
\begin{gathered}
E\left(L, T \mid B_{0}\right)=E_{\min }(L)+4 \delta \epsilon_{1} L(1-L \delta)+\delta^{2} \sum_{k=1}^{L}\left(8 \epsilon_{1}-4 \varepsilon(k)\right)(L-k) \sigma_{k}, \\
S\left(L, T \mid B_{0}\right)=\int_{0}^{T} \frac{1}{T} d E\left(L, T \mid B_{0}\right) \\
=\delta L \frac{4 \epsilon_{1}+T}{T}-\delta^{2} L^{2} \frac{8 \epsilon_{1}+T}{2 T}+\delta^{2} \sum_{k=1}^{L} \frac{8 \epsilon_{1}-4 \varepsilon(k)+T}{T}(L-k) \sigma_{k} .
\end{gathered}
$$

ii) Much less probable configuration of the borders is $B_{1, k}$ with only one symbol -1 at distance $k$ from the edge of segment $M$. The corresponding probability does not depend on $k$ and is approximately equal to $\delta, P\left(B_{1, k}\right) \approx \delta$. For this configuration, the conditional total energy and entropy are:

$$
\begin{gathered}
E\left(L, T \mid B_{1, k}\right)=E_{\min }(L)+2 \sum_{j=1}^{L} \varepsilon(k+j-1)+\delta \sum_{j=1}^{L}\left(4 \epsilon_{1}-4 \varepsilon(k+j-1)\right) \sigma_{k+j-1}, \\
S\left(L, T \mid B_{1, k}\right)=\int_{0}^{T} \frac{1}{T} d E\left(L, T \mid B_{1, k}\right)=\delta \sum_{j=1}^{L} \frac{4 \epsilon_{1}-4 \varepsilon(k+j-1)+T}{T} \sigma_{k+j-1} .
\end{gathered}
$$

iii) Probabilities of all other configurations of the borders are negligible with respect to $P\left(B_{1, k}\right)$. There is no need to find the corresponding conditional energies and entropies if we are interested in the first non-additive corrections to these quantities.

Averaging the conditional quantities over border configurations $B_{0}$ and $B_{1, k}$ and keeping the non-additive temperature dependent terms in the main approximations in $\delta$, we derive the actual quantities $E(L, T)$ and $S(L, T)$ :

$$
\begin{gathered}
E(L, T)=E_{\min }(L)+4 \delta\left(\epsilon_{1} L+\sum_{r=1}^{N} r \varepsilon(r)\right) \\
S(L, T)=L \delta\left[\frac{4 \epsilon_{1}}{T}(1-2 \delta)+1+\delta \frac{4 H-T}{2 T}+\delta \sum_{k=1}^{N} \frac{8 \epsilon_{1}-4 \varepsilon(k)+T}{T}\left(\sigma_{k}-1\right)\right] \\
+\delta^{2}\left[\sum_{k=1}^{N} k\left(\sigma_{k}-1\right)-\frac{4}{T} \sum_{k=1}^{N} k \varepsilon(k) \sigma_{k}\right] .
\end{gathered}
$$

Similar calculations for the internal energy give the following expression:

$$
E_{M}^{\mathrm{in}}(L, T)=E_{\min }^{\mathrm{in}}(L)+4 \delta\left(\epsilon_{1} L-\sum_{r=1}^{N} r \varepsilon(r)\right) .
$$




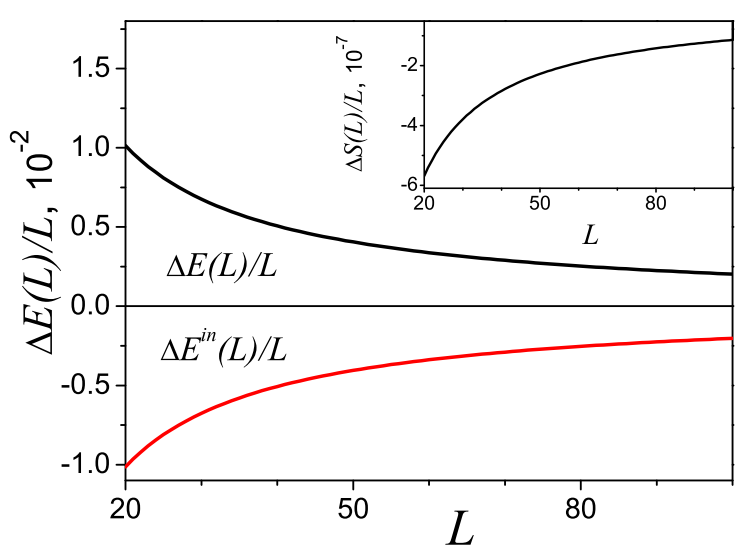

FIG. 3: (Color online) The specific non-additive energies $E, E^{\text {in }}$ and entropy $S$ versus the length $L$ of a segment for step-like function $\varepsilon(r)=1 / 20, \quad r \leq 20$. Symbol $\Delta$ denotes the non-additive terms in Eqs. (39), (41) and (42). Other parameters are: $T=3, H=6$.

One can see that the non-additive total energy and entropy, determined by Eqs. (39) and (41), do not satisfy the relation $d E(L, T)=T d S(L, T)$, unlike the conditional total energy and entropy. The dependences of the non-additive energy and entropy on $L$ are presented in Fig. 3 for a step-like function $\varepsilon(r)$.

As mentioned above, in the absence of an external thermostat, the temperature $T$ of a chain is controlled by its initial state. In this case, the temperature can be expressed via the total energy of the chain. In particular, the positive initial energy of the chain provides negative temperature (see Fig. 4). If the initial energy is close to its maximum, the temperature is negative and close to zero and the system is almost "frozen". The lesser the positive energy the lower the negative temperature. If the initial energy tends to zero, the chain becomes more chaotic and its temperature tends to the minus infinity.

The situation is principally different for the chains in contact with an external thermostat. The states of chain with negative temperatures become unstable and, therefore, do not exist. If the initial energy of the chain is negative and close to zero, the temperature is positive and high. At the same time, it tends to zero for the energy close to its minimum. Both with and without the external thermostat, all the statistical properties of the chain with positive temperature are the same. 


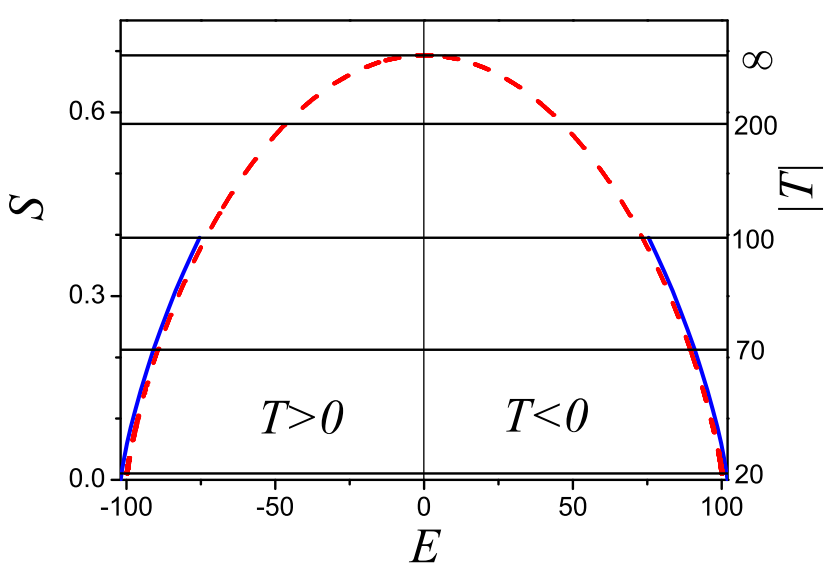

FIG. 4: (Color online) The dependence of the entropy $S$ on the total energy $E$ for the step-like function $\varepsilon(r)=1 / 20, \quad r \leq 20$. Magnetic field $H=100$ is so strong that the high-temperature (red dashed curve), Eqs. (29), (33), and low-temperature (blue solid curve), Eqs. (39), (41), asymtotes coincide. The corresponding values of the temperature are shown on the right scale.

\section{CONCLUSION}

Thus, we have studied the statistical properties of mesoscopic segments of Ising spin chains with finite but arbitrary long interaction ranges. The equivalence of the Ising and $N$-step Markov chains was used for calculating the averaged statistical quantities. In particular, the averaged magnetization in the presence of the external magnetic field and its RMS were calculated in the two limiting cases of strong and weak interactions. Correlations in the chain result in the non-additive behavior of these quantities as the segment length $L$ increases. We show that the statistical quantities of the chain can be obtained by averaging the corresponding conditional quantities. The explicit expressions for the non-additive energy, internal energy, and entropy are derived in the limiting cases of high and low temperatures comparing to the energy of spin interaction. At high temperatures, the equilibrium Ising chain of spin turns out to be equivalent to the additive multi-step Markov chain.

[1] Dynamics and Thermodynamics of Systems with Long-Range Interactions, T. Dauxois et. al., Lecture Notes in Physics Vol. 602 (Springer-Verlag, New York, 2002).

[2] T. Padmanabhan, Phys. Rep. 188, 285 (1990). 
[3] D.R. Nicholson, Introduction to Plasma Theory (John Wiley, New York, 1983).

[4] J. Barre, T. Dauxois, G. De Ninno, D. Fanelli, and S. Ruffo, Phys. Rev. E 69, 045501(R) (2004).

[5] F. Baldovin and E. Orlandini, Phys. Rev. Lett. 97, 100601 (2006).

[6] G. K. Zipf, Human Behavior and the Principle of Least Effort (Addison-Wesley, New York, 1949).

[7] S. S. Melnyk, O. V. Usatenko, V. A. Yampol'skii, and V. A. Golick, Phys. Rev. E 72, 026140 (2005).

[8] K. E. Kechedzhy, O. V. Usatenko, and V. A. Yampol'skii, Phys. Rev. E 72, 046138 (2005).

[9] Z. Ouyang, C. Wang, and Z.-S. She, Phys. Rev. Lett. 93, 078103 (2004).

[10] N. Sourlas, Nature (London) 339, 693 (1989).

[11] R. N. Mantegna, H. E. Stanley, Nature (London) 376, 46 (1995).

[12] D. Poland and H. Scheraga, J. Chem. Phys. 45, 1456 (1966).

[13] R. Everaers, S. Kumar, C. Simm, Phys. Rev. E 75, 041918 (2007).

[14] F. J. Dyson, Commun. Math. Phys., 12, 91 (1969); F. J. Dyson, Commun. Math. Phys., 12, 212 (1969); F. J. Dyson, Commun. Math. Phys. 21, 269 (1971).

[15] D. Ruelle, Commun. Math. Phys. 9, 267 (1968).

[16] D. J. Thouless, Phys. Rev. 187, 732 (1969).

[17] C. Tsalis, J. Stat. Phys. 52, 479 (1988).

[18] L. Casetti, M. Kastner, Phys. Rev. Lett. 97, 100602 (2006).

[19] O. V. Usatenko and V. A. Yampol'skii, Phys. Rev. Lett. 90, 110601 (2003); O. V. Usatenko, V. A. Yampol'skii, K. E. Kechedzhy and S. S. Melnyk, Phys. Rev. E 68, 061107 (2003).

[20] S. S. Melnyk, O. V. Usatenko, V. A. Yampol'skii, S. S. Apostolov, Z. A. Mayselis, J. Phys. A: Math. Gen. 39, 14289 (2006).

[21] S. S. Apostolov, Z. A. Mayzelis, O. V. Usatenko, V. A. Yampol'skii, arXiv:physics/0610081, to be published in Int. J. Mod. Phys. B.

[22] S. S. Apostolov, Z. A. Mayzelis, S. S. Melnyk, O. V. Usatenko, V. A. Yampol'skii, Physica A 376, 165 (2007).

[23] Z.A. Mayzelis, S. S. Apostolov, S. S. Melnyk, O. V. Usatenko, V. A. Yampol'skii, Chaos, Solitons and Fractals 34, 112 (2007).

[24] Statistical Physics: An Advanced Approach with Applications, J. Honerkamp (Springer-Verlag, 
1998).

[25] J. Besag, J. Royal Stat. Soc. B 36, 192 (1974).

[26] S. S. Apostolov, Z. A. Mayzelis, O. V. Usatenko, and V. A. Yampol'skii, Europhys. Lett. 76 (6), 1015 (2006).

[27] Metropolis N. et. al., J. Chem. Phys. 21, 1087 (1953).

[28] S. S. Melnyk, O. V. Usatenko, V. A. Yampol'skii, Physica A 361, 405 (2005). 